\title{
Theoretical Study of the Seasonal Behavior of the Global Ionosphere at Solar Maximum
}

\author{
J. J. Sojka And R. W. Schunk \\ Center for Atmospheric and Space Sciences, Utah State University, Logan
}

\begin{abstract}
A time-dependent, three-dimensional, multi-ion numerical model of the global ionosphere was used to study the asymmetry in large-scale ionospheric features between the northern and southern hemispheres. The comparisons were done for June and December solstice conditions at solar maximum for quiet geomagnetic activity. Simple conditions and diurnally reproducible ionospheric features were established in order to elucidate the intrinsic hemispherical differences that are associated with the different displacements between the geomagnetic and geographic poles and the different atmospheric conditions. In comparing the ionospheric densities in the northern and southern hemispheres for a given season, we found the following: (1) The winter hemispheres display the most marked universal time (UT) variations due to the displacement between the geomagnetic and geographic poles, (2) The summer high-latitude and equatorial densities in both hemispheres are morphologically similar. However, there is a factor of 2 difference in Appleton anomaly densities in certain places between the June and December solstices due to the seasonal dependence of the atmospheric densities, (3) In the winter hemispheres, the corresponding electron densities are again morphologically similar but can be very different quantitatively due to the different dipole offsets, (4) For a given season, the density difference between the northern and southern hemispheres displays a marked UT dependence. For similar winter conditions, the densities in the northern and southern hemispheres can differ by an order of magnitude in places at certain UTs, (5) The winter mid-latitude trough is the feature that exhibits the largest northernsouthern hemisphere difference due to the different dipole tilts, and (6) The "winter anomaly" is present in the northern hemisphere at almost all UTs, while it is essentially absent in the southern hemisphere. In the northern hemisphere, the anomaly maximizes between 1000 and $1600 \mathrm{UT}$, with the winter/summer peak electron density $\left(N_{m} F_{2}\right)$ ratio reaching 1.6. In our model, the anomaly results primarily from the adopted atmospheric densities.
\end{abstract}

\section{INTRODUCTION}

Over the past decade, significant advancement in the observation and modeling of the northern hemisphere ionosphere has been made. The strong ionospheric universal time (UT) (diurnal) dependence has been predicted and observed in different ways. This effect, which arises due to the displacement between the geographic and geomagnetic poles, is expected to be even more significant in the southern hemisphere where the displacement is considerably larger. In our first study using a global ionospheric model [Sojka and Schunk, 1985], we considered June solstice conditions and were able to show significant hemispherical asymmetries in the global $F$ region. However, because only one solstice condition was considered, we were unable to compare the two hemispheres for the same seasonal conditions.

In this paper we have used our global model of the $F$ region to carry out a seasonal study. This study is aimed at providing a state-of-the-art global scale comparison of the northern and southern ionosphere based upon a global physical model of the ionosphere. These data can then form a basis against which comparisons with future conjugate observations can be made. Our model is a time-dependent, threedimensional, multi-ion model of the global ionosphere at altitudes between 120 and $800 \mathrm{~km}$ [cf. Schunk and Walker, 1973; Schunk et al., 1975, 1986; Schunk and Raitt, 1980; Sojka et al., 1981a; Schunk and Sojka, 1982]. The model takes

\footnotetext{
Copyright 1989 by the American Geophysical Union.
}

$P_{\text {ap }}$

per number 88JA04202.

8-0227/89/88JA-04202\$05.00 account of field-aligned diffusion, cross-field electrodynamic drifts both in the equatorial region and at high latitudes, interhemispheric flow, thermospheric winds, polar wind escape, energy-dependent chemical reactions, neutral composition changes, ion production due to solar EUV radiation and auroral precipitation, thermal conduction, diffusionthermal heat flow, and local heating and cooling processes. Our model also takes account of the offset between the geomagnetic and geographic poles and the bending of field lines near the magnetic equator.

The model studies were carried out for both June and December solstice conditions at solar maximum and for low geomagnetic activity. In section 2 we describe our global $F$ region model and the inputs that we adopted for this particular study. The global $F$ region densities calculated with our model are presented in section 3 , and in section 4 we summarize our results.

\section{Global $F$ Region Model}

\subsection{F Region Model}

The ionospheric model was initially developed as a midlatitude, multi-ion $\left(\mathrm{NO}^{+}, \mathrm{O}_{2}^{+}, \mathrm{N}_{2}^{+}\right.$, and $\left.\mathrm{O}^{+}\right)$model by Schunk and Walker [1973]. The time-dependent ion continuity and momentum equations were solved as a function of altitude for a corotating plasma flux tube including diurnal variations and all relevant $E$ and $F$ region processes. This model was extended to include high latitude effects due to convection electric fields and particle precipitation by Schunk et al. $[1975,1976]$. A simplified ion energy equation was also added, which was based on the assumption that local heating and cooling processes dominate (valid below 500 
$\mathrm{km})$. Flux tubes of plasma were followed as they moved in response to convection electric fields. A further extension of the model to include the minor ions $\mathrm{N}^{+}$and $\mathrm{He}^{+}$, an updated photochemical scheme, and the MSIS atmospheric model is described by Schunk and Raitt [1980].

The addition of plasma convection and particle precipitation models is described in Sojka et al. [1981a, b]. More recently, the ionospheric model has been extended by Schunk and Sojka [1982] to include ion thermal conduction and diffusion-thermal heat flow, so that the ion temperature is now rigorously calculated at all altitudes between 120 and $1000 \mathrm{~km}$. The adopted ion energy equation and conductivities are those given by Conrad and Schunk [1979]. Also, the electron energy equation has been included recently by Schunk et al. [1986], and consequently, the electron temperature is now rigorously calculated at all altitudes. The electron energy equation and the heating and cooling rates were taken from Schunk and Nagy [1978], and the conductivities were taken from Schunk and Walker [1970]. The incorporation of the Sterling et al. [1969] equatorial ionospheric model and the various improvements to this model are described in Sojka and Schunk [1985].

\subsection{Combined Global Model}

The global model consists of two separate ionospheric simulation models, namely, a mid- and high-latitude formulation and an equatorial formulation. The difficulties associated with joining the two models are described by Sojka and Schunk [1985]. This paper will be referred to for the details concerning inputs as well as $F$ region results when they complement this seasonal study.

One of the major sources of asymmetry between the hemispheres is the magnetic field topology relative to the earth's rotational axis. This effect was found to introduce major UT variations, which were different in the two hemispheres. For the first study, we adopted a north magnetic pole located at $78.6^{\circ} \mathrm{N},-69.8^{\circ} \mathrm{E}$ and a south magnetic pole located at $-75.5^{\circ} \mathrm{N}$ and $126^{\circ} \mathrm{E}$ geographic. These locations were based upon the work of Mead [1970] and the auroral observations of Bond [1968]. Since publishing our first global paper in 1985, Fraser-Smith [1987] has extensively reviewed the question of centered and eccentric dipole fields. Fraser-Smith shows how centered and eccentric dipoles can be derived from the International Geomagnetic Reference Field (IGRF) coefficients. For the northern hemisphere, our adopted centered dipole is within $1 / 2^{\circ}$ of latitude and $1^{\circ}$ of longitude of the centered dipole axis computed by Fraser-Smith. In the southern hemisphere, our axis agrees to within $3 / 4^{\circ}$ in latitude and $7^{\circ}$ in longitude to the location of the eccentric axial pole location computed by Fraser-Smith. Our equatorial model uses an eccentric dipole defined by the intersections of these two magnetic poles with the Earth's surface. Again, this is in close agreement with the work of Fraser-Smith. This is especially the case when the resolution of our global model, $3^{\circ}$ latitude and $15^{\circ}$ in longitude for this study, is considered.

In the first part of our study, where the global seasonal/UT effects are considered, the effect of changing the dipole tilt angle is shown. To do this, additional southern hemisphereionospheric simulations were performed with the dipole tilt angle being increased from 14.5 to 19.5 . This $5^{\circ}$ additional shift, although somewhat arbitrary, does demonstrate the ionospheric UT dependence upon the magnetic pole loca- tion. It is also in the direction of the south magnetic dip pole, which is at a geographic latitude of approximately $-67^{\circ}$. The importance of a dip pole is found in computing induced (via neutral winds or electric fields) vertical drifts which drastically affect $N_{m} F_{2}, h_{m} F_{2}$, and the topside ionosphere. Other than this additional magnetic dipole config. uration, the mid-high latitude and equatorial formulations are the same as described by Sojka and Schunk [1985].

\subsection{Global Inputs}

This study complements our earlier global study carried out for one solstice condition, namely, June solstice. Hence we present in this paper results from both June and December solstice to show seasonal effects in the global ionosphere. The specific solar conditions are solar maximum, defined by an $F 10.7$ value of 170 , and low geomagnetic activity, defined by a $K p=2$ and $A p=12$. Several other inputs are required: (1) equatorial electric potential; (2) high latitude electric potential; (3) global auroral precipitation; (4) global neutral atmosphere; and (5) global neutral winds. Each of these model inputs is described by Sojka and Schunk [1985]. Only a brief description is given here, since they are identical to those used in this earlier global study.

The adopted equatorial electric potential is from Richmond et al. [1980]. To incorporate this into our model, we followed the method used by Sterling et al. [1969] in which a set of UT-independent cosine functions are described by a set of parameters. These functions and parameters are then related to the Richmond et al. [1980] data set. The electric field extends about the geomagnetic equator, to $\pm 30^{\circ}$ latitude. At the magnetic equator, the corresponding induced vertical $\mathbf{E} \times \mathbf{B}$ drifts range between $\pm 11 \mathrm{~m} / \mathrm{s}$. The high latitude electric potential corresponds to a symmetric two-cell pattern of the Volland [1978] type. The specific form of the electric field is given by Sojka et al. [1980]. The total crosspolar potential is $48 \mathrm{kV}$, with uniform sun-aligned potentials in the polar cap and a potential that decreases as the inverse of sine colatitude to the fourth power equatorward of the polar cap. This symmetric pattern is shifted by $1.5^{\circ}$ in the antisunward direction from the magnetic pole in both hemispheres. In our global model the equatorial, high-latitude, and corotational potentials are added, and the resulting potentials are then used to compute the electric fields.

The adopted global auroral precipitation model is based on the empirical model of Spiro et al. [1982] for low geomagnetic activity conditions, $K p \sim 2$. In our model, the empirical values of energy flux are used to scale a characteristic auroral ion production profile. The production profile is taken from Knudsen et al. [1977] and corresponds to auroral precipitation of $2-\mathrm{keV}$ characteristic energy. These production rates and those for solar EUV are dependent upon the global neutral atmosphere. The mass/spectrometer incoherent scatter (MSIS) model atmosphere [Hedin et al., 1977] is used for this purpose.

For the purpose of defining a neutral wind, the coordinate system whose equatorial plane contain the subsolar point is adopted. In this frame, the neutral wind is assumed to be longitudinally symmetric about $0100-1300$ LT (see Figure 7 in Sojka and Schunk [1985]). On the dayside, the maximum poleward drift is $50 \mathrm{~m} / \mathrm{s}$, while in the dark polar cap the wind reaches $200 \mathrm{~m} / \mathrm{s}$ in an antisunward direction. Only the meridional component of this wind is used. It is 


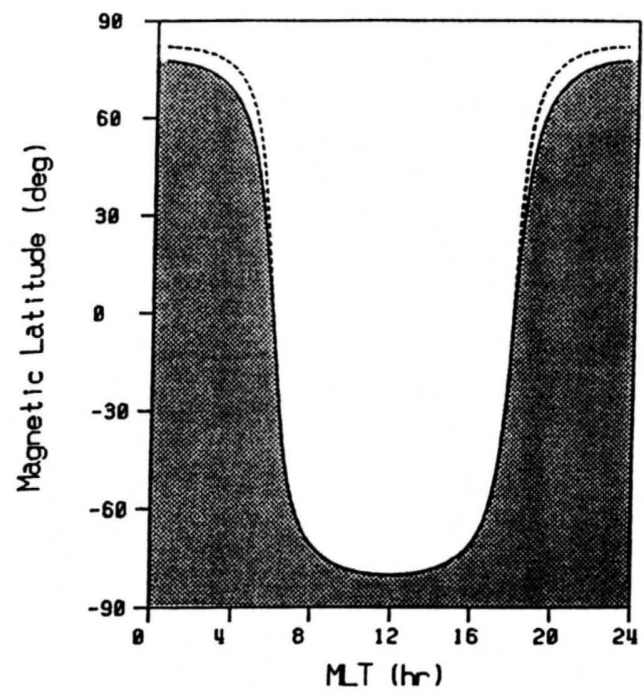

Fig. 1a. Terminator location in a magnetic latitude-MLT reference frame for summer solstice at 0700 UT (solid line). The shading indicates the dark regions. The dashed line is the terminator location for winter solstice at $1900 \mathrm{UT}$, but the latitudinal scale has been inverted to aid the comparison.

transformed to the magnetic frame, in which the vertical induced drift is computed. Although the wind is symmetric in the special subsolar frame, in the ionospheric simulation the induced vertical drift does not show a large hemispherical asymmetry. This neutral wind pattern has been adopted; it has not been computed self-consistently with the particular solar and ionospheric conditions.

In looking at the seasonal effects associated with the ionosphere, we have kept the above inputs constant. The major difference between the two solstice conditions is the change in solar illumination and, consequently, EUV ion production when viewed in either the geographic or magnetic frame. This effect is further heightened by the eccentric nature of the dipole axis, leading to significant hemispherical differences. Figure $1 a$ shows the terminator location in the magnetic frame for June solstice at 0700 UT. The northern polar region is entirely sunlit, while the southern region is dark. During December solstice, 12 hours later, one expects a similar illumination in the magnetic frame, but with inverted latitudes, i.e., dark in the north and the south sunlit. The dashed line indicates the "inverted latitude" terminator location for December solstice at 1900 UT. Note the two terminator curves are not symmetric about the equator, which is due to the noncentered nature of the dipole axis. On a global scale the difference between these two terminator curves does not seem large. However, as will be seen, it is significant. For the extra dipole tilt case $\left(19.5^{\circ}\right)$, the difference in terms of ionospheric effects is even more marked. Figure $1 b$ repeats the terminator comparison for the other UT phase, 1900 UT in June solstice (solid line) and 0700 UT in December solstice (dashed line). Comparing Figures $1 a$ and $1 b$ clearly indicates why the UT effect is so marked in the winter high latitude regions.

\section{F Region Densities}

The model described in the previous sections was run for solar maximum, June solstice, and low magnetic activity conditions. At middle and high latitudes, all trajectories were started from noon steady state density profiles. Flux tubes were followed for times in excess of 24 hours, determined by the time the slowest flux tube takes to convect once around its trajectory [see Sojka et al., 1981a]. All trajectories were followed 12 times, at 2-hour UT intervals, so that UT effects could be taken into account. After a complete traversal of a trajectory, the difference between the final density profile obtained and the starting noon steady state profile was negligible for latitudes greater than about $20^{\circ}$. A similar procedure to that described above was used for the equatorial model, except that all flux tubes were followed for 48 hours and only the densities obtained during the second 24-hour period were kept. This additional run time was necessary in order to obtain a repeatable density profile after a complete traversal of a trajectory.

As each flux tube is run, altitude or flux tube profiles are stored. These profiles consist of $\mathrm{NO}^{+}, \mathrm{O}_{2}^{+}, \mathrm{N}_{2}^{+}, \mathrm{O}^{+}, \mathrm{N}^{+}$, and $\mathrm{He}^{+}$at 4 -km intervals from 120 to $800 \mathrm{~km}$ for the midhigh latitude model, and $\mathrm{NO}^{+}, \mathrm{O}_{2}^{+}, \mathrm{N}_{2}^{+}, \mathrm{O}^{+}$, and $\mathrm{N}^{+}$at a variable altitude interval for the equatorial model. In the equatorial bottomside $F$ region, the step size is less than 4 $\mathrm{km}$, while above $800 \mathrm{~km}$ the step size is greater than $10 \mathrm{~km}$. Such profiles are stored every $0.5^{\circ}$ to $2^{\circ}$ in longitude (local time). The final data set consists of ion densities binned into $3^{\circ}$ magnetic latitude, one-hour MLT, and 2-hour UT intervals.

\subsection{Global $N_{e}$ at $300 \mathrm{~km}$}

Figure $2 a$ displays the electron density at $300 \mathrm{~km}$ for one particular geometry of solar illumination in the magnetic local time frame. The solar illumination corresponds to that shown in Figure 1b. In the left panels, the 1900 UT, June solstice conditions are shown, while the right panels correspond to $0700 \mathrm{UT}$, December solstice conditions plotted with an inverted latitudinal axis. The smaller panels show only the effect of increasing the dipole tilt in the southern hemisphere from $14.5^{\circ}$ to $19.5^{\circ}$. In Figure $2 a$, the larger left panel corresponds to the conditions of our first global study [Sojka

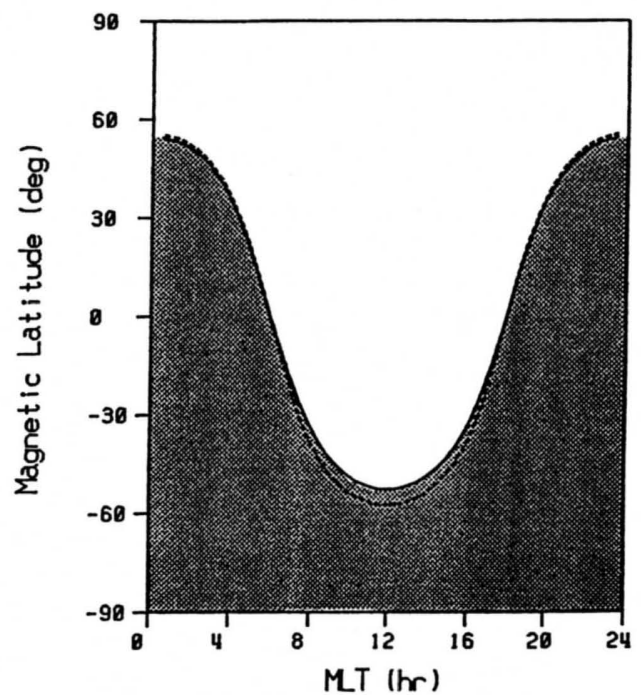

Fig. 1b. Terminator location in a magnetic latitude-MLT reference frame for summer solstice at 1900 UT (solid line). The dashed line is the terminator location for winter solstice at 0700 UT, but the latitudinal scale has been inverted to aid the comparison. 


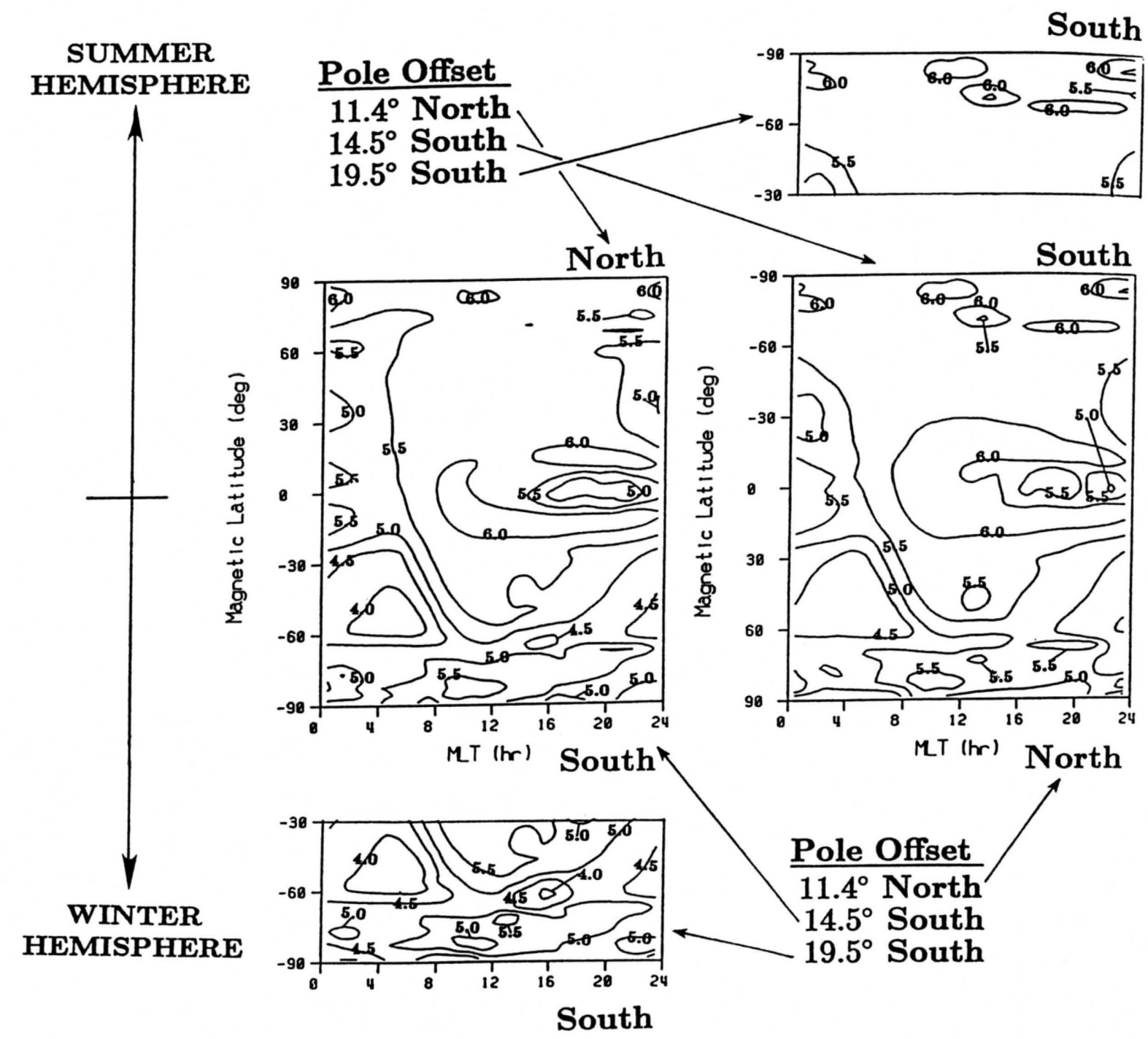

Fig. 2a. Global plot of the electron density variation at $300 \mathrm{~km}$ in a magnetic latitude-MLT reference frame. The left panels are for June solstice at $1900 \mathrm{UT}$ and the right panels are for December solstice at 0700 UT. The top half of the figure is for the summer hemisphere and the bottom half is for the winter hemisphere. The two smaller panels show the $N_{e}$ variation at $300 \mathrm{~km}$ in the southern hemisphere for a larger offset between the magnetic and geographic poles. Note that the UTs selected are the same as for Figure $1 b$, which shows the region of darkness. The $N_{e}$ contours are labeled in $\log _{10} N_{e}\left(\mathrm{~cm}^{-3}\right)$.

and Schunk, 1985]. For that study, we displayed $N_{m} F_{2}$ in a color format [see plate 1 in Sojka and Schunk, 1985]. These densities at $300 \mathrm{~km}$ differ somewhat from the $N_{m} F_{2}$ values, however the overall features are similar.

A number of distinct $F$ region features can be seen in Figure $2 a$ (upper left panel), which divide the $F$ region into three primary latitudinal regions. The Appleton peaks lie on either side of the magnetic equator at approximately $\pm 10^{\circ}$ and are present from about noon until 0400 MLT. For solstice conditions, these peaks are asymmetric due primarily to the effect of the neutral wind. At mid-latitudes, from $20^{\circ}$ to $60^{\circ}$, the most distinct feature is the nocturnal midlatitude trough. In the winter hemisphere, the density minimum is approximately an order of magnitude lower than in the summer hemisphere. The morning local time boundary of this trough shows the season dependence of the terminator. In the two polar regions poleward of about $\pm 60^{\circ}$, the $F$ region is highly dependent upon magnetospheric processes. Ion production due to auroral precipitation and magneto- spheric convection causes the polar cap to appear highly complex. In the summer hemisphere, where the polar cap is sunlit, the weak auroral precipitation associated with the low geomagnetic activity conditions is insufficient to create a marked auroral oval, whereas in the winter hemisphere, very distinct auroral oval and convection features are present.

This global morphology can be compared with that obtained for December solstice by comparing in Figure $2 a$ the left larger panel with the right larger panel. The comparison is aided by the right panel inverted latitude scale, which causes summer to be in the top and winter in the bottom part of the panel. Summer high latitude densities in both hemispheres are morphologically identical, with densities ranging from $4 \times 10^{5}$ to $10^{6} \mathrm{~cm}^{-3}$. Also, the equatorial regions are very similar, with densities ranging from less than $10^{5}$ to greater than $10^{6} \mathrm{~cm}^{-3}$. The slight difference in the Appleton anomaly between the June and December solstice is of the order of a factor of 2 in places and is associated with the seasonal dependence in MSIS rather than "dipole 
SUMMER HEMISPHERE

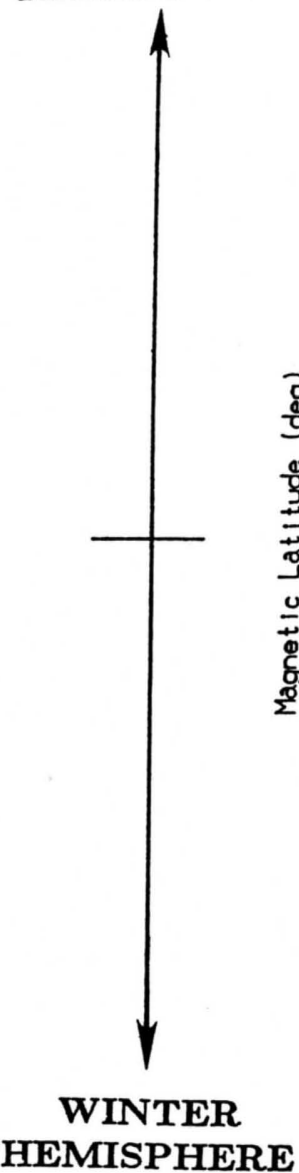

Pole Offset $11.4^{\circ}$ North 14.5 South $\backslash$ $19.5^{\circ}$ South

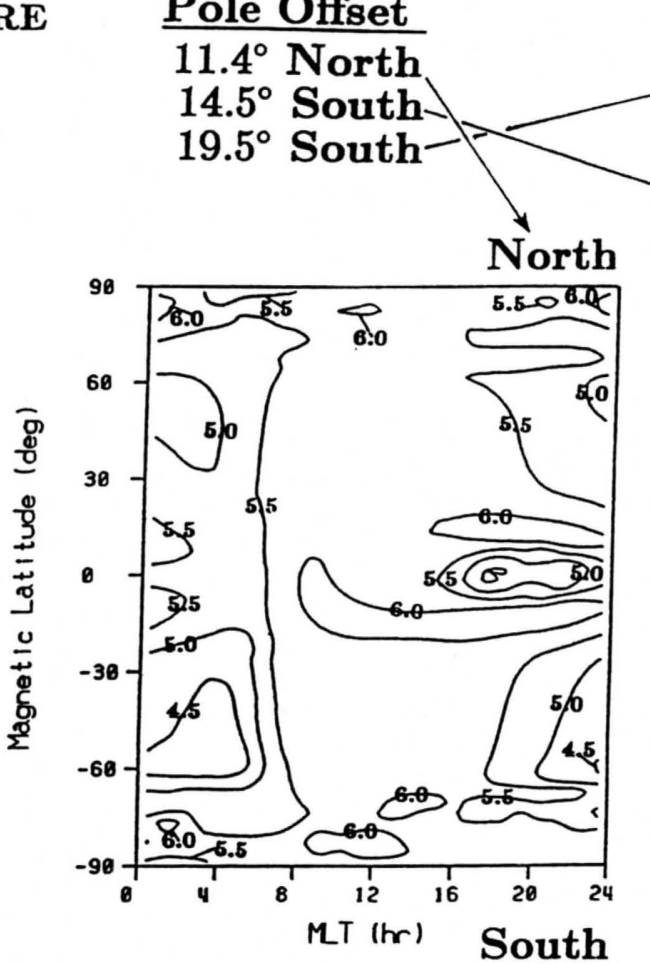

MT (tor)

South
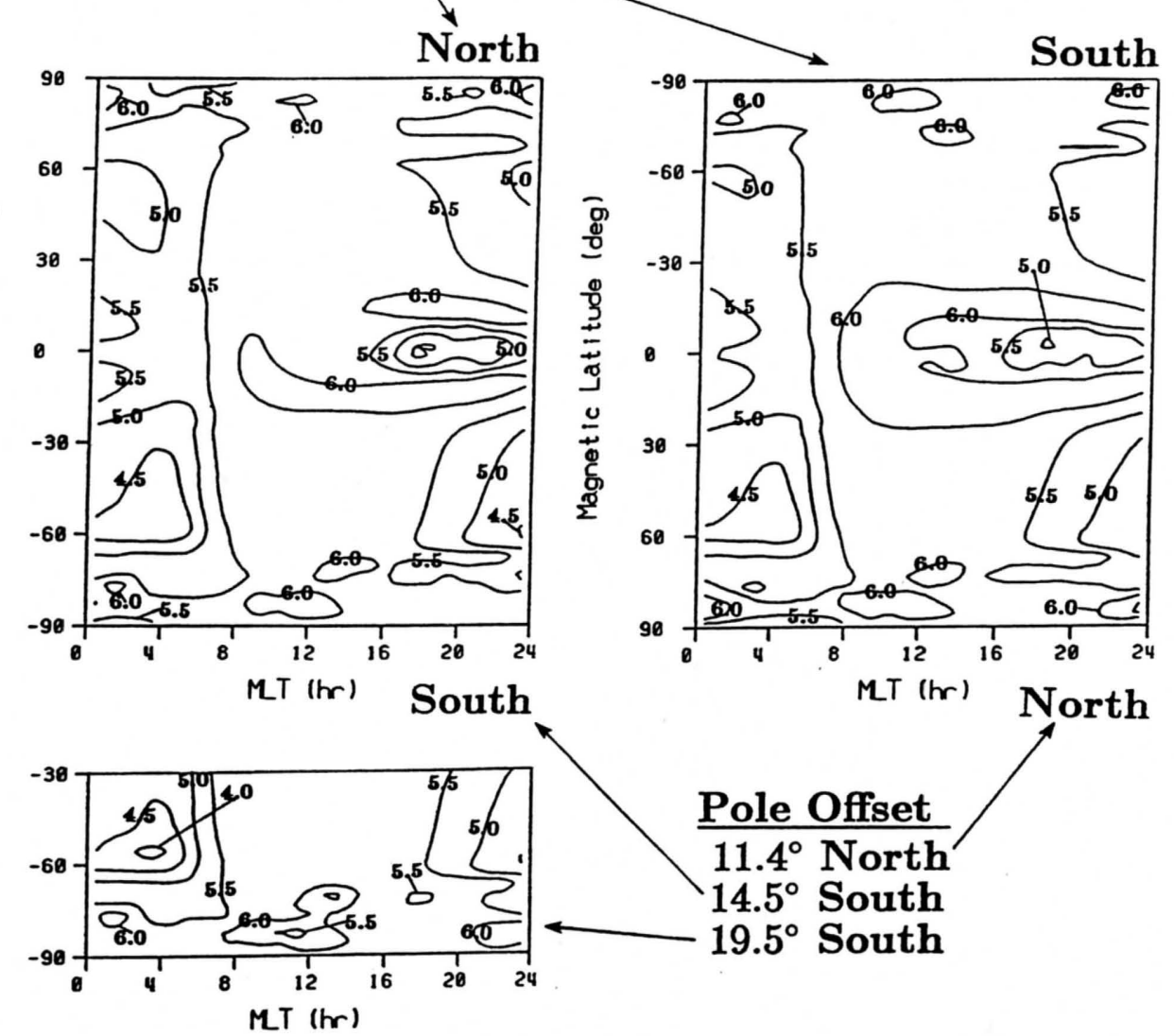

South

Fig. 2b. Global plot of the $N_{e}$ variation at $300 \mathrm{~km}$ in a magnetic latitude-MLT reference frame. The left panels are for June solstice at 0700 UT and the right panels are for December solstice at 1900 UT. The top half of the figure is for the summer hemisphere and the bottom half is for the winter hemisphere. The two smaller panels show the $N_{e}$ variation in the southern hemisphere for a larger offset between the magnetic and geographic poles. Note that the UTs selected are the same as for Figure 1a, which shows the region of darkness.

tilt" effects. In the winter hemisphere, the two solstice conditions are again morphologically similar, but quite different quantitatively. The early morning trough is about twice as deep in the southern hemisphere. In the region between the dayside oval and the sunlit terminator, the density drops below $3 \times 10^{4} \mathrm{~cm}^{-3}$ in the southern hemisphere, but only to about $7 \times 10^{4} \mathrm{~cm}^{-3}$ in the northern hemisphere. These differences in both the nocturnal and dayside trough are due to the different dipole tilts in the two hemispheres. This is further demonstrated in the lower left panel, where the southern winter hemisphere is shown for the case of a $19.5^{\circ}$ tilt. In this case, the dayside trough density is further decreased by a factor of 3 to 4 . For these extreme winter UT conditions, the entire auroral-polar ionosphere is in darkness, and hence this region shows almost no hemispherical difference. Similarly, in the summer hemisphere (top panels) and the extra southern hemisphere $19.5^{\circ}$ offset case (upper right panel) when the oval and polar regions are entirely il- luminated, the hemispherical differences due to the different dipole tilts are negligible.

Figure $2 b$ shows the $N_{e}$ variation in the magnetic local time frame for the solar illumination conditions shown in Figure 1a. In the summer hemisphere (upper panels), the density variations are similar to those in Figure $2 a$, even the absolute densities are within a factor of about 2 . The trough boundaries have changed somewhat due to the different terminator locations. Densities in the equatorial regions at this UT, which is 12 hours different from the Figure $2 a$ UT, are almost identical. However, in the winter hemisphere, the densities are quite different from those at a UT 12 hours different. The dayside trough region no longer exists, and instead of densities less than $2 \times 10^{4}$ the densities are greater than $4 \times 10^{5} \mathrm{~cm}^{-3}$. Because of partially being sunlit at this UT, the polar regions have significantly higher densities, about a factor of 4 to 10 greater. The winter hemisphere shows the most marked seasonal and UT de- 
SUMMER
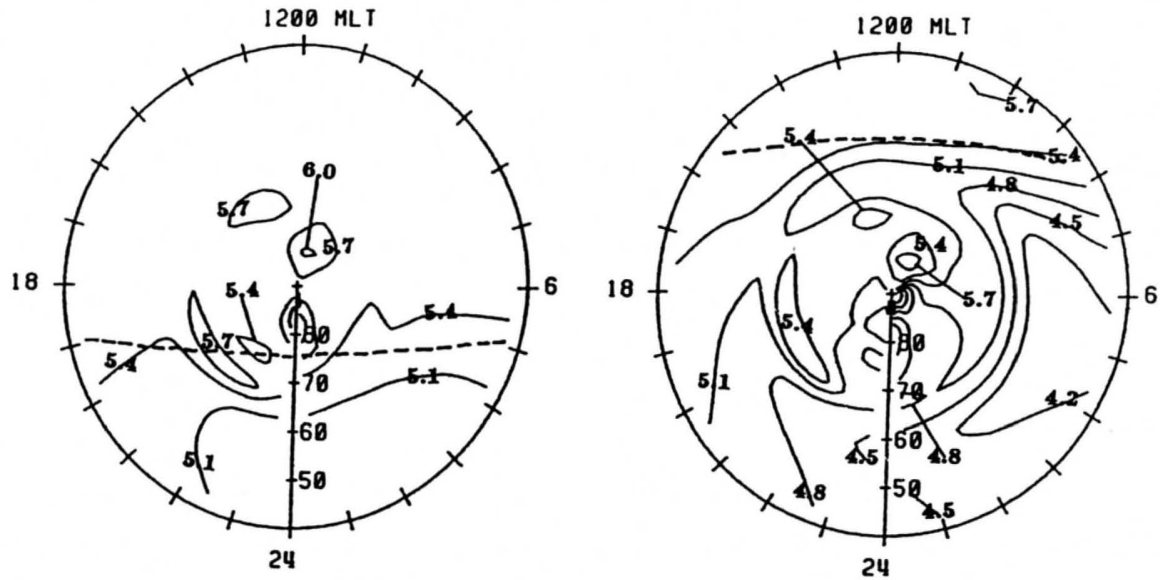

SUMMER

WINTER
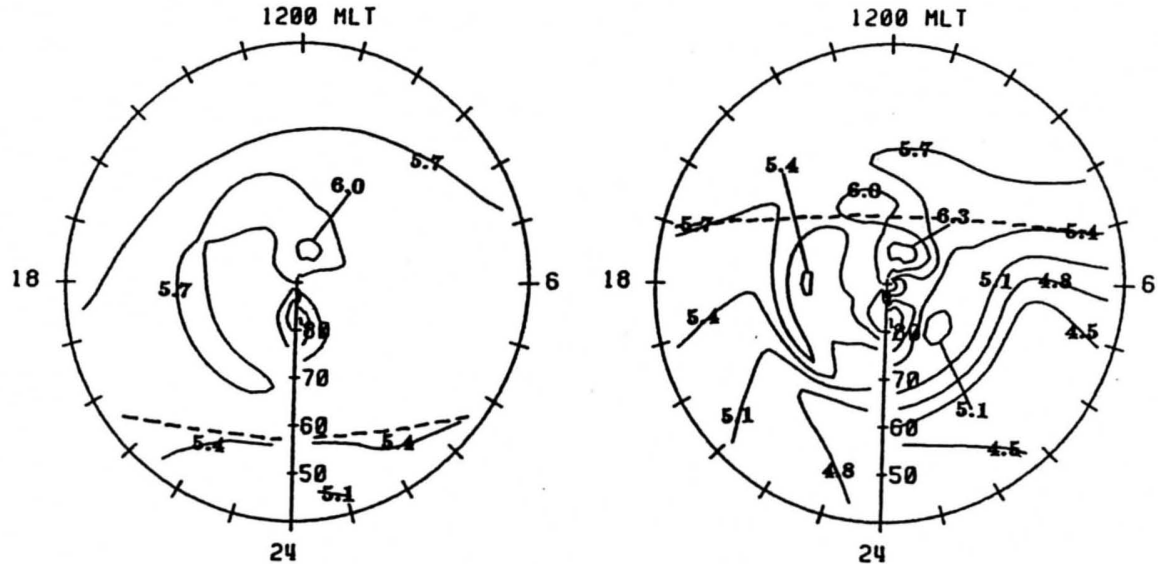

Fig. 3a. Seasonal variation of $N_{e}$ at $300 \mathrm{~km}$ shown in a magnetic latitude MLT reference frame for both the northern hemisphere (top panels) and the southern hemisphere (bottom panels) at $0700 \mathrm{UT}$.

pendencies arising from the differences in dipole offset. At a higher resolution, the north and south hemispheres show density differences of the order of a few tens of percent due to differences in the MSIS atmospheric densities between the hemispheres.

\subsection{High-Latitude Seasonal Trends}

To look at the polar regions in more detail, we change from a rectangular to a polar diagram presentation. This also allows for an easier comparison with earlier studies. In our earlier studies of the high latitude ionosphere, we have shown how various ionospheric features, such as the polar hole, tongue of ionization, night sector trough, and nightside auroral densities, develop depending on the geophysical conditions [Sojka et al., 1981a, b, c; Sojka and Schunk, 1983]. In the earlier global study [Sojka and Schunk, 1985], we modeled June solstice conditions and compared the southern hemisphere winter UT morphology with the northern hemisphere summer UT morphology. We now focus specifically on the northern and southern hemisphere differences for the same season and use a dipole tilt of $14.5^{\circ}$ for the southern hemisphere.

Figure $3 a$ shows the comparison of $N_{e}$ at $300 \mathrm{~km}$ for summer (left panels) and winter (right panels) for the same UT, namely, $0700 \mathrm{UT}$. The top two panels correspond to the northern hemisphere, while the bottom two panels correspond to the southern hemisphere. In terms of snapshots, the June solstice condition corresponds to the top left and bottom right panels, while the December solstice condition corresponds to the top right and bottom left panels. The summer hemispheres at 0700 UT look similar with densities ranging between $3 \times 10^{5}$ to about $10^{6} \mathrm{~cm}^{-3}$. A night sector trough region, with densities of the order of $10^{5} \mathrm{~cm}$, is present in both hemispheres. However, the latitudinal and MLT extent of the trough are defined by the particular terminator location (dashed lines). The summer ionosphere has densities within a factor of 2 for the northern and southern hemispheres.

In the winter panels (right side), the densities show a wider range of variation from about $10^{4}$ to greater than $10^{6}$ $\mathrm{cm}^{-3}$. From the terminator locations, it is evident that at this UT the northern hemisphere auroral and polar regions are in darkness, whereas in the southern hemisphere the terminator extends into the noon sector oval and polar cap. This leads to the northern hemisphere winter densities being lower than the corresponding southern hemisphere values. This was also the case for the summer conditions, where the southern hemisphere densities were up to a fac- 
SUMMER

WINTER

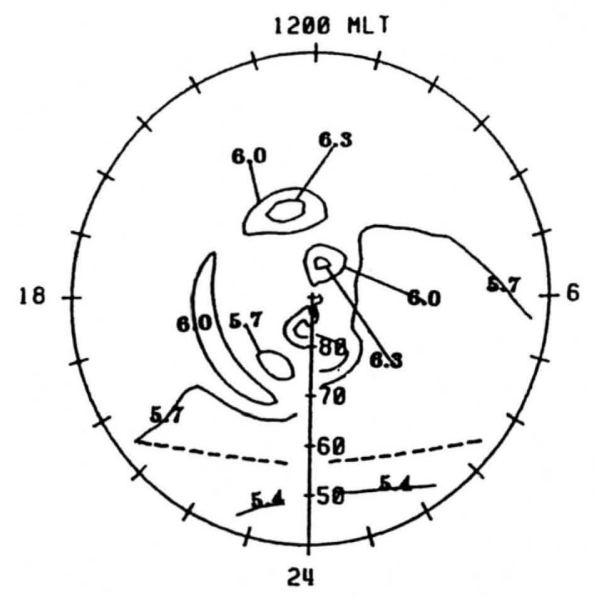

!

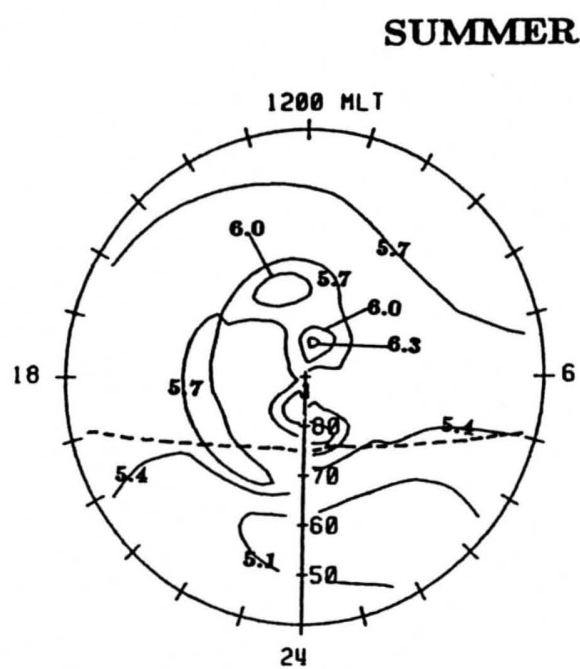

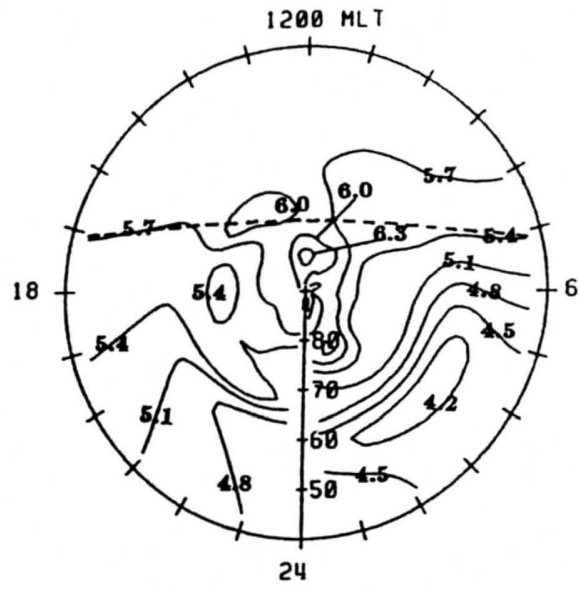

WINTER

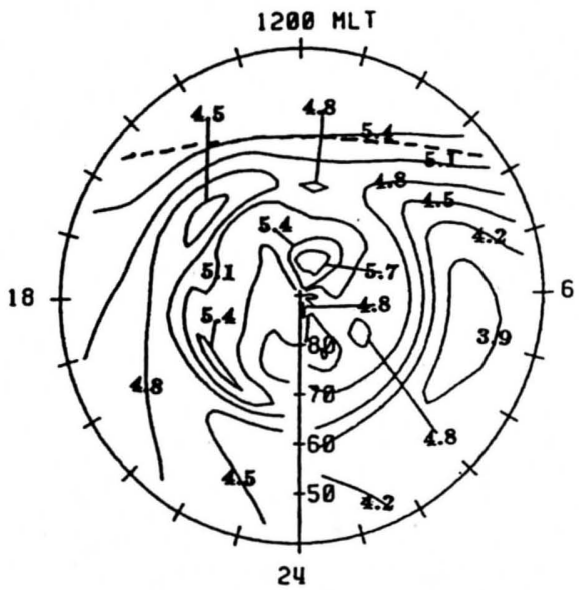

Fig. 3b. Seasonal variation of $N_{e}$ at $300 \mathrm{~km}$ shown in a magnetic latitude-MLT reference frame for both the northern hemisphere (top panels) and the southern hemisphere (bottom panels) at $1900 \mathrm{UT}$.

tor of 2 higher. In winter, the difference is significantly larger. In the noon auroral and polar regions, the density difference is of the order of a factor of 5 . The northern hemisphere has a region of lower densities $\left(\sim 10^{5} \mathrm{~cm}^{-3}\right)$ between the solar illuminated dayside and the auroral oval. The plasma convecting into the polar cap has a low density, and hence the tongue of ionization is a relatively low density feature $\left(\lesssim 4 \times 10^{5} \mathrm{~cm}^{-3}\right)$. The southern hemisphere, on the other hand, is sunlit in this region, the depletion is not present, and the tongue of ionization reaches densities of about $2 \times 10^{6} \mathrm{~cm}^{-3}$. In both hemispheres, the density of the tongue maximizes in the noon sector near the magnetic pole due to the induced upward $\mathbf{E} \times \mathbf{B}$ drift. Since this study is for low geomagnetic activity, the auroral precipitation is weak ( $\lesssim 0.33 \mathrm{erg} \mathrm{cm}^{-2} \mathrm{~s}^{-1}$ ) and consequently, the auroral oval is only slightly discernable. However, in this region the densities are within a factor of 2 for the two hemispheres as well as for the summer-winter comparison. The night sector mid-latitude trough is significantly more extensive in latitude and magnetic local time in winter than in summer. Again, the northern hemisphere trough is about a factor of 2 deeper than in the southern hemisphere as well as being extended to later times in the morning sector $(\sim 0900$ MLT), whereas in the southern hemisphere it only extends to about 0600 MLT.

Figure $3 b$ is identical to Figure $3 a$, except that now the electron densities are plotted at $1900 \mathrm{UT}$. At this UT, the orientation of the magnetic and geographic poles relative to the sun has been reversed. The summer hemisphere electron densities (left panels) in sunlight range from $4 \times 10^{5}$ to $2 \times 10^{6}$ $\mathrm{cm}^{-3}$. This range is very similar to that at 0700 UT (Figure $3 a$, left panels). Also, the trough densities are of the order of $1.2 \times 10^{5} \mathrm{~cm}^{-3}$, again similar to those at $0700 \mathrm{UT}$. In this case, the southern hemisphere densities are lower than the corresponding northern hemisphere densities by about a factor of 2 .

The right panels of Figure $3 b$ show the winter comparison, which shows hemispheric differences greater than an order of magnitude in places. This difference is significantly greater than the 0700 UT winter differences. A major feature not present at $0700 \mathrm{UT}$ is the postnoon trough minimum in the southern hemisphere at 1300-1400 MLT. At 1900 UT, this 
minimum density drops below $3 \times 10^{4} \mathrm{~cm}^{-3}$, whereas the northern hemisphere minimum density at this location and $0700 \mathrm{UT}$ is only $1 \times 10^{5} \mathrm{~cm}^{-3}$ and at $1900 \mathrm{UT}$ it is about $1 \times 10^{6} \mathrm{~cm}^{-3}$. This feature indicates how the hemispheres can be asymmetric, with density differences of factors of 2 to 4 due to the different magnetic field geometries in the two hemispheres. In winter the UT variation is of the order of 2 to 10 , while in summer it is only about a factor of 2 . The UT variation is 12 hours shifted in phase between the two hemispheres, but is significantly different in the two hemispheres. This can be further seen by looking at the post midnight mid-latitude trough minimum in winter. In the northern hemisphere, the minimum density varies between $1.0 \times 10^{4}$ and $1.5 \times 10^{4} \mathrm{~cm}^{-3}$, whereas in the southern hemisphere the variation is between $5 \times 10^{3}$ and $2 \times 10^{4} \mathrm{~cm}^{-3}$. The auroral precipitation regions are, however, not (in our model) sensitive to UT variations. What UT differences exist are associated with the differences in the solar terminator location or changes in the neutral atmosphere between the two hemispheres. At $1900 \mathrm{UT}$, the polar cap densities, especially the tongue of ionization, are very similar to the corresponding 0700 UT densities.

\subsection{Trough Morphology}

In the previous section a major asymmetry between the two hemispheres was found to be associated with the winter mid-latitude trough. Figure 4 shows the trough morphology for six UTs for both the northern and southern winter hemispheres. The electron density at $300 \mathrm{~km}$ is contoured and shaded in the panel to emphasize the trough location and depth. Gray areas correspond to regions where the densities are less than $5.0 \times 10^{4} \mathrm{~cm}^{-3}$ and black corresponds to densities less than $2.5 \times 10^{4} \mathrm{~cm}^{-3}$. Each panel is associated with a specific UT, which ranges from $0100 \mathrm{UT}$ at the top to $2100 \mathrm{UT}$ at the bottom. The left and right panels are for the southern and northern hemisphere, respectively. The mid-latitude trough is most extensive at $1700 \mathrm{UT}$ and 0500 UT for the southern and northern hemispheres, respectively. This 12-hour phase shift is also applicable to the least extended situation; 0900 UT in the southern and 2100 UT in the northern hemisphere. Beyond this difference, it is clear. that the UT variation of the trough extent is different in the two hemispheres by as much as 4 hours in MLT; i.e., the maximum dusk sector extent of the trough at $2100 \mathrm{UT}$ in the southern hemisphere and the corresponding northern trough at $1300 \mathrm{UT}$. In summer, the differences are still present; however, both the extent and depth of the midlatitude trough are significantly restricted, as seen in Figure 2.

\section{Seasonal Anomaly}

The mid-latitude dayside ionosphere exhibits a well defined seasonal variation in $N_{m} F_{2}$. Torr and Torr [1973] extensively documented this variation on a global scale. It is both hemispherical and longitude (UT) dependent. In the northern hemisphere, the winter $N_{m} F_{2}$ densities systematically exceed those in summer, leading to the terminology "seasonal" or "winter" anomaly. In contrast, the southern hemisphere exhibits predominantly a summer (or equinox) $N_{m} F_{2}$ density peak. Several basic mechanisms have been proposed to describe this anomalous and hemispherical dependence [Torr and Torr, 1973; Torr et al., 1980]. The winter anomaly is primarily associated with the enhancement in the neutral $[\mathrm{O}] /\left[\mathrm{N}_{2}\right]$ ratio in winter. In the south. ern hemisphere, its absence is tenuously associated with a geomagnetic activity energy source which, due to the significantly different magnetic field topology, is able to affect the thermosphere preferentially in this hemisphere. Additional thermospheric chemistry processes have been proposed to account for the fine details of the anomaly (see Torr et al. [1980] for a review of these). In our global model, we have the thermospheric properties described by the MSIS model, but do not have either the detailed magnetic field topology nor the complex chemistry schemes. Hence our seasonal anomaly results are primarily from the neutral atmosphere global and seasonal variations.

For the first time we are in a position to look at the seasonal anomaly on a global scale with our ionospheric model. Global winter and summer densities for identical geomagnetic conditions have been calculated. Using these densities, ratios of $N_{m} F_{2}$ for winter and summer have been computed in order to determine the effect of the winter anomaly. In Figure 5 this ratio is plotted as a function of magnetic latitude along the noon magnetic meridian for six different UTs. The left panel corresponds to the northern hemisphere, while the right panel corresponds to the southern hemisphere. These ratios, in addition to showing a UT variation, show an even stronger hemispherical difference. Whereas the northern hemisphere shows a winter anomaly at almost all UTs from $30^{\circ}$ to $65^{\circ}$ magnetic latitude, the southern hemisphere shows no anomaly. In the northern hemisphere, the anomaly maximizes between 1000 and $1600 \mathrm{UT}$ at a value of 1.6 , while from 0200 to $0800 \mathrm{UT}$ the anomaly is almost not present with ratios ranging from 0.4 to 1.0 . These lower than unity ratios correspond to UTs when the winter dayside trough is present and clearly indicates a significant reduction in winter $N_{m} F_{2}$ primarily due to the absence of EUV production. In the southern hemisphere, the same effect occurs between 1400 and 2000 UT when the southern hemisphere dayside trough is present.

The difference between the two hemispheres is not specifically related to the magnetic field differences. In the case of this major anomaly difference, the source of the difference lies in the neutral atmosphere. MSIS [Hedin et al., 1977] is responsible for the primary longitudinal and hemispherical differences present in Figure 5. This hemispherical anomaly morphology is to zeroth-order consistent with observations. Torr and Torr [1973] have collected and presented an extensive review of anomaly data which indicates the following: (1) The anomaly in the northern hemisphere is significantly more marked than in the southern hemisphere where it is almost entirely absent; and (2) In the northern hemisphere, a longitudinal (UT) variation is present which gives the largest anomaly values around 1200 UT. These zerothorder findings are the same as our model results, although the longitudinal phase in the northern hemisphere is different. Since our global chemistry scheme contains only the dominant chemical reactions [Schunk and Raitt, 1980], this anomaly agreement is quite remarkable. Improved agreement would require a more complex chemistry scheme, including metastable chemistry, and possibly also transport. In order to investigate the longitudinal phase relationship in more detail, a significantly more detailed study would have to be undertaken, which is beyond the scope of the current work. 

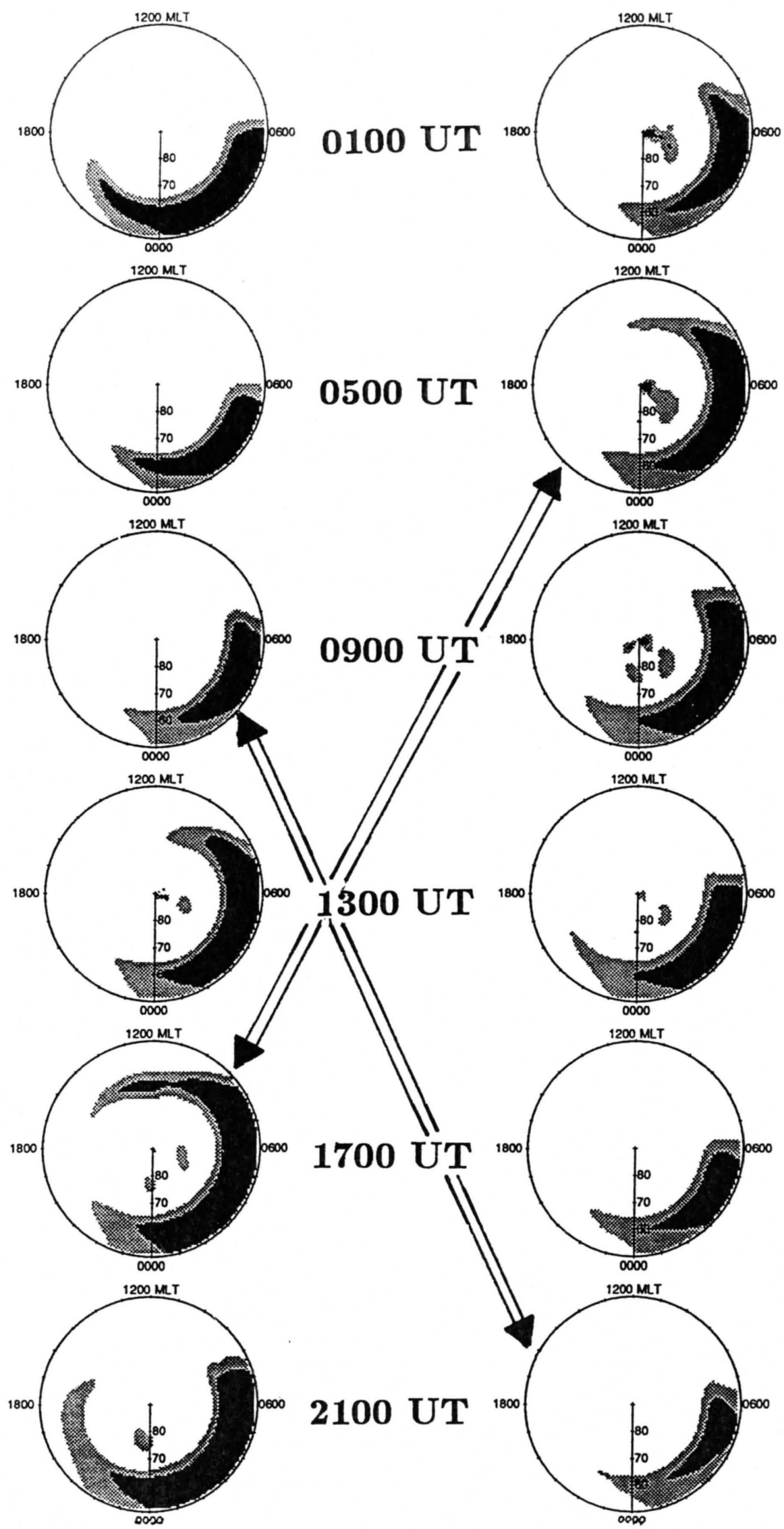


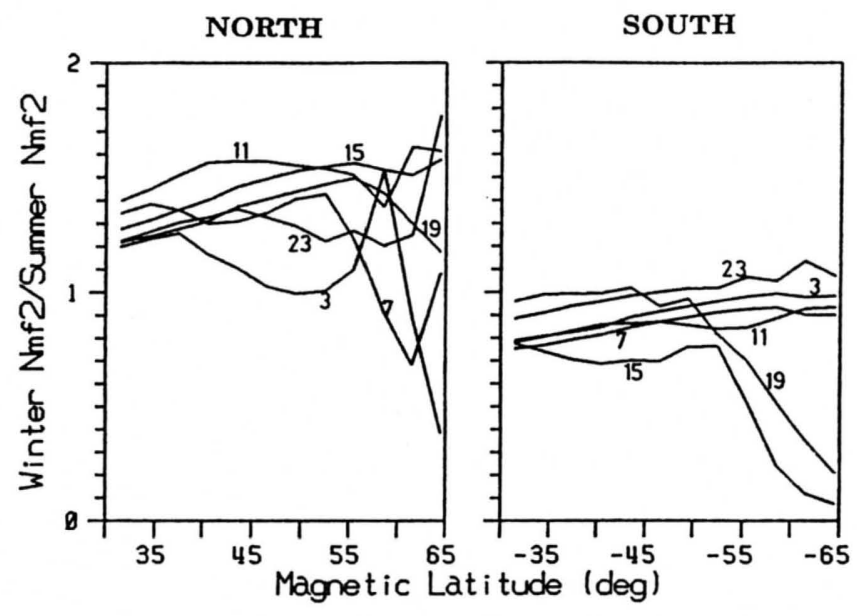

Fig. 5. The ratio of winter/summer $N_{m} F_{2}$ values along the noon meridian as a function of magnetic latitude. The left panel is for the northern hemisphere and the right panel is for the southern hemisphere. Each curve corresponds to a different UT and is labeled in hours.

\section{Summary AND Discussion}

A time-dependent, three-dimensional, multi-ion numerical model of the global ionosphere was used to study the asymmetry in large-scale ionospheric features between the northern and southern hemispheres. The comparisons were done for both June and December solstice conditions at solar maximum for quiet geomagnetic activity. Diurnally reproducible conditions were established in order to elucidate the intrinsic hemispherical differences that are associated with the different displacements between the geomagnetic and geographic poles and the different atmospheric conditions, as specified by the MSIS model.

Various inputs are needed for our global model, including the neutral temperature, composition and wind; the electric field distribution; the auroral precipitation pattern; the solar EUV spectrum; and a magnetic field model. Since these inputs are needed over the entire globe at all times, it was necessary to adopt empirical models for the inputs. For this study we used the MSIS model for the neutral densities and temperatures, a simple analytical function to describe the global wind pattern, a Volland [1978] two-cell convection pattern to describe high-latitude electrodynamics, the Richmond et al. [1980] equatorial electric field model, the Spiro et al. [1982] empirical model of auroral precipitation, and a dipole magnetic field model [Fraser-Smith, 1987].

With regard to the inputs, it should be noted that the comparison of the northern and southern hemispheres could be done for an infinite number of geophysical conditions. Comparisons could be done for different seasonal, solar cycle, and interplanetary magnetic field (IMF) conditions as well as for different magnetic activity levels. Comparisons could also be done for magnetic storms and substorms, during which the magnetospheric inputs vary markedly with time. However, in this initial comparison, we attempted to keep the geophysical conditions as simple as possible in order to clearly identify intrinsic hemispherical differences. Therefore we adopted quiet geomagnetic conditions so that atmospheric dynamics would not have an appreciable effect on our results. Also, the Volland convection pattern was taken to be symmetric with a cross-tail potential of $48 \mathrm{kV}$, and a low level of auroral precipitation was adopted $(K p=2$,
$A p=12)$. In addition, the convection and precipitation patterns were assumed to be identical in the northern and southern hemispheres, and these inputs were kept fixed so that diurnally reproducible ionospheric densities could be calculated. In the future, hemispherical comparisons will be conducted for more complicated geophysical conditions.

The main results of our study were displayed at two universal times, 0700 and 1900 UT. These times correspond to the extremes of solar illumination in the polar regions at the solstices. Specifically, at June solstice solar illumination maximizes at $1900 \mathrm{UT}$ in the northern polar region, while at December solstice it maximizes at 0700 UT in the southern polar region. With this fact in mind, our study indicates the following:

1. At both UTs, the $F$ region is divided into three primary latitudinal regions; an equatorial region, where the Appleton ionization peaks dominate from about noon until 0400 MLT; a mid-latitude region, where the "main" or "mid-latitude" trough is the dominant feature; and a polar region, where the density variation is large in winter.

2. The weak auroral precipitation associated with quiet magnetic activity is insufficient to create a marked auroral oval in the summer hemisphere, where the overall density level is high due to solar production, but it is sufficient to produce a marked oval in the winter hemisphere, where the background electron densities are an order of magnitude lower.

3. The summer high-latitude densities in both hemispheres are morphologically similar, with the densities ranging from $4 \times 10^{5}$ to $10^{6} \mathrm{~cm}^{-3}$. The equatorial densities are also similar in the summer hemispheres, with the densities ranging from less than $10^{5} \mathrm{~cm}^{-3}$ to greater than $10^{6} \mathrm{~cm}^{-3}$. However, there is a factor of 2 difference in the Appleton anomaly densities in certain places between the June and December solstices. This difference is associated with the seasonal dependence in MSIS rather than dipole tilt effects.

4. In the winter hemispheres, the corresponding electron densities are again morphologically similar, but very different quantitatively. For example, the early morning trough is about twice as deep in the winter southern hemisphere than in the winter northern hemisphere. This difference is due to the different dipole tilts in the two hemispheres.

5. The winter hemispheres display the most marked UT variations due to the displacement between geomagnetic and geographic poles.

6 . For a given season, the density "difference" between the northern and southern hemispheres displays a marked UT dependence. For example, when the hemispheres are compared for similar winter conditions, the density difference is greater than an order of magnitude in places at certain UTs.

7. The tongues of ionization that extend across the polar cap in both hemispheres in winter are qualitatively similar, but they can be quantitatively different at certain UTs due to the different dipole offsets.

8. The winter mid-latitude trough is the feature that exhibits the largest northern-southern hemisphere difference due to the different dipole tilts.

9. The winter anomaly is present in the northern hemisphere at almost all UTs from $30^{\circ}$ to $65^{\circ}$ magnetic latitude, while it is essentially absent in the southern hemisphere. In the northern hemisphere, the anomaly maximizes between 1000 and $1600 \mathrm{UT}$, with the winter/summer $N_{m} F_{2}$ density ratio reaching 1.6. In our model, the MSIS atmospheric den- 
sities are the primary cause of the winter anomaly, and the different dipole tilts in the two hemispheres play a secondary role.

As noted throughout the paper, the ionospheric features predicted by our global model are in qualitative agreement with the available measurements. However, we have not attempted to do a detailed comparison between model predictions and observations owing to the lack of comprehensive global data sets for both June and December solstice and quiet geomagnetic activity. For a meaningful quantitative comparison, we need data from satellites and several groundbased stations suitably located throughout the world. Because of the marked UT variation of the ionosphere, the data need to be taken simultaneously for at least a 24-hour period. In addition to $F$ region densities and temperatures, we also need the inputs to our model (neutral wind, auroral precipitation, magnetospheric and equatorial electric potential patterns, etc.). Unfortunately, at the present time, the existing data base is far too incomplete to warrant a detailed quantitative comparison between model predictions and observations of characteristic features in the northern and southern hemispheres.

Acknowledgments. This research was supported by AFOSR contract F49620-86C-0109, NASA grant NAGW-77, and NSF grant ATM-8417880 to Utah State University. The computer modeling effort was supported by the National Center for Atmospheric Research, which is sponsored by the National Science Foundation.

The Editor thanks G. J. Bailey and B. J. Watkins for their assistance in evaluating this paper.

\section{REFERENCES}

Bond, F. R., Magnetic and auroral conjugacy, Ann. Geophys., 24, 1-7, 1968.

Conrad, J. R., and R. W. Schunk, Diffusion and heat flow equations with allowance for large temperature differences between interacting species, J. Geophys. Res., 84, 811-822, 1979.

Fraser-Smith, A. C., Centered and eccentric geomagnetic dipoles and their poles, 1600-1985, Rev Geophys., 25, 1-16, 1987.

Hedin, A. E., C. A. Reber, G. P. Newton, N. W. Spencer, H. C. Brinton, H. G. Mayr, and W. E. Potter, A global thermospheric model based on mass spectrometer and incoherent scatter data, MSIS 2, Composition, J. Geophys. Res., 82, 2148, 1977.

Knudsen, W. C., P. M. Banks, J. D. Winningham, and D. M. Klumpar, Numerical model of the convecting $F_{2}$ ionosphere at high latitudes, J. Geophys. Res., 82, 4784-4792, 1977.

I lead, G. D., International geomagnetic reference field 1965.0 in dipole coordinates, J. Geophys. Res., 75, 4372-4374, 1970.

R.chmond, A. D., M. Blanc, B. A. Emery, R. H. Wand, B. G. Fejer, R. F. Woodman, S. Ganguly, P. Amayenc, R. A. Behnke, C. Calderon, and J. V. Evans, An empirical model of quietday ionospheric electric fields at middle and low latitudes, $J$. Geophys. Res., 85, 4658-4664, 1980.

Schunk, R. W., and A. F. Nagy, Electron temperatures in the $F$ region of the ionosphere: Theory and observations, Rev. Geophys., 16, 355-399, 1978.
Schunk, R. W., and W. J. Raitt, Atomic nitrogen and oxygen ions in the daytime high-latitude $F$ region, J. Geophys. Res., $85,1255-1272,1980$.

Schunk, R. W., and J. J. Sojka, Ion temperature variations in the daytime high-latitude $F$ region, $J$. Geophys. Res., 87, 5169$5183,1982$.

Schunk, R. W., and J. C. G. Walker, Transport properties of the ionospheric electron gas, Planet. Space Sci., 18, 1535-1550, 1970.

Schunk, R. W., and J. C. G. Walker, Theoretical ion densities in the lower ionosphere, Planet. Space Sci., 21, 1875-1896, 1973.

Schunk, R. W., W. J. Raitt, and P. M. Banks, Effect of electric fields on the daytime high-latitude $E$ and $F$ regions, $J$. Geophys. Res., 80, 3121-3130, 1975.

Schunk, R. W., P. M. Banks, and W. J. Raitt, Effect of electric fields and other processes upon the nighttime high latitude $F$ layer, J. Geophys. Res., 81, 3271-3282, 1976.

Schunk, R. W., J. J. Sojka, and M. D. Bowline, Theoretical study of the electron temperature in the high-latitude ionosphere for solar maximum and winter conditions, J. Geophys. Res., 91, 12041-12045, 1986.

Sojka, J. J., and R. W. Schunk, A theoretical study of the highlatitude $F$ region's response to magnetospheric storm inputs, J. Geophys. Res., 88, 2112-2122, 1983.

Sojka, J. J., and R. W. Schunk, A theoretical study of the global $F$ region for June solstice, solar maximum, and low magnetic activity, J. Geophys. Res., 90, 5285-5298, 1985.

Sojka, J. J., W. J. Raitt, and R. W. Schunk, A plasma convection model, Rep. Cent. for Atmos. and Space Sci., Utah State Univ., Logan, 1980.

Sojka, J. J., W. J. Raitt, and R. W. Schunk, A theoretical study of the high-latitude winter $F$ region at solar minimum for low magnetic activity, J. Geophys. Res., 86, 609-621, 1981a.

Sojka, J. J., W. J. Raitt, and R. W. Schunk, Theoretical predictions for ion composition in the high-latitude winter $F$ region for solar minimum and low magnetic activity, J. Geophys. Res., $86,2206-2216,1981 b$.

Sojka, J. J., W. J. Raitt, and R. W. Schunk, Plasma density features associated with strong convection in the winter highlatitude $F$ region, J. Geophys. Res., ,86, 6968-6976, $1981 c$.

Spiro, R. W., P. H. Reiff, and L. J. Maher, Precipitating electron energy flux and auroral zone conductances: An empirical model, J. Geophys. Res., 87, 8215-8227, 1982.

Sterling, D. L., W. B. Hanson, R. J. Moffett, and R. G. Baxter, Influence of electromagnetic drifts and neutral air winds on some features of the $F_{2}$ region, Radio Sci., 4, 1005-1023, 1969.

Torr, D. G., P. G. Richards, and M. R. Torr, Ionospheric composition: The seasonal anomaly explained, paper presented at Symposium on "The Physical Basis of the Ionosphere in the SolarTerrestrial System," Pozzuoli, Italy, AGARD/NATO, 1980.

Torr, M. R., and D. G. Torr, The seasonal behaviour of the F2 layer of the ionosphere, J. Atmos. Terr. Phys., 35, 2237-2251, 1973.

Volland, H., A model of the magnetospheric electric convection field, J. Geophys. Res., 83, 2695-2699, 1978.

R. W. Schunk and J. J. Sojka, Center for Atmospheric and Space Sciences, Utah State University, Logan, UT 84322.

(Received September 6, 1988; revised November 28, 1988; accepted December 1, 1988.) 